\title{
THE IMPLICIT PATH COST OPTIMIZATION IN DIJKSTRA ALGORITHM USING HASH MAP DATA STRUCTURE
}

\author{
Mabroukah Amarif and Ibtusam Alashoury \\ Department of Computer Sciences, Sebha University, Sebha, Libya
}

\begin{abstract}
The shortest path between two points is one of the greatest challenges facing the researchers nowadays. There are many algorithms and mechanisms that are designed and still all according to the certain approach and adopted structural. The most famous and widely used algorithm is Dijkstra algorithm, which is characterized by finding the shortest path between two points through graph data structure. It's obvious to find the implicit path from the solution path; but the searching time varies according to the type of data structure used to store the solution path. This paper improves the development of Dijkstra algorithm using linked hash map data structure for storing the produced solution shortest path, and then investigates the subsequent implicit paths within this data structure. The result show that the searching time through the given data structure is much better than restart the algorithm again to search for the same path.
\end{abstract}

\section{KEYWORDS}

Dijkstra algorithm, data structure, linked hash map, time complexity, implicit path, graph

\section{INTRODUCTION}

The graph is defined as a set of nodes (Vertices) and edges that link these nodes. Each edge is marked with a weight value describing the cost between the connected nodes. There are two types of graph; directed graph for which each node is directed by one way to any other node, and undirected graph for which its possible to go to and back from the same way between two connected nodes. The issue of the shortest path problem is related to graph theory, which is one of the most important topics for researchers [1, 2, 3, 4, 5, 6, 7]. It concerns with finding the shortest path between two nodes, or between a node as a source to all other nodes, depending on the weights of the edges that link these nodes [8]. The graph theory and shortest path are used widely especially in the practical applications of various fields, the most important are roads, transport networks and geographic information systems $[9,10,11,12]$.

Various algorithms have been created to find the shortest path within graphs. These algorithms depend on the graph and path type. The most famous of these algorithms are Dijkstra algorithm [13], Bellman, the Johnson, and the Floyd Warshall algorithms [14].

Based on the rapid development and handling of the huge amount of road networks data and their development methods, researchers try to improve the previous algorithms or develop their own $[4,7,9,10,11,12]$. They are also exploring more various ways and methods to get the lowest cost of path into a large number of nodes and speeding up their search process. Some of them are working on reconstructing the graphs in an attempt to reduce the cost of searching time either by 
compression, optimization or subgraphs $[1,2,3,4,5,6,15]$. Others are replacing data structure with another $[18,19,20,21]$.

However, the field remains open to researchers to discover more ways and mechanisms in an attempt to get the lowest search cost within a large number of nodes and accelerate the search process which is a challenge until this time. This paper proposes an improvement of Dijkstra algorithm using a special data structure (linked hash map) for storing the solution path (shortest path given by Dijkstra algorithm) to optimize the searching time for the implicit paths. The proposed algorithm uses Dijkstra algorithm with priority queue implemented by min heap to find the solution path (shortest path). The solution path is stored into data structure of array list contains of a linked hash map elements. We have tested the proposed algorithm with different graphs sizes up to 10000 nodes. The following section describes the most related works to our paper while section 3 explains the algorithm description. Analysis and results are described in section 4. A discussion of this paper is explained in section 5 and the conclusion is provided in section 6 .

\section{RELATED WORK}

Dijkstra algorithm was designed by the Dutch computer scientist Edsger Dijkstra in 1956 and published in 1959 [13]. It's the most popular algorithm in the area of finding shortest path from single source to a node destination, or multiple nodes destination within a graph $[16,17,22]$. It can also be used to find the shortest route costs from the source to the destination by stopping the algorithm once the shortest route is set to the specific target.

This algorithm has been considered by many researchers to improve the shortest path cost by minimizing the searching time (time complexity) using different data structure. Jain et. al. improve the Dijkstra algorithm by using priority queue and linked list [18]. It has been noticed that by using a graph represented by thier adjacncey lists and the priority queue implemented as a min-heap, the time efficiency is in $\mathrm{O}(|\mathrm{E}| \log |\mathrm{V}|)$, where $\mathrm{V}$ is the number of nodes and $\mathrm{E}$ is the number of edges which connected these nodes. if the priority queue is implemented using an advanced data structure called the Fibonacci heap, the time becomes $\mathrm{O}(|\mathrm{V}| \log \mathrm{V}+\mathrm{E})$, and its imporved [16].

Time efficiency could be imporved by exploit the solution path to get the implicit paths if they are queried again. From the literature, most algorithms may provide this feature, but there is no such explanation or imporvement of it. A suitable data structure could improve the time efficiency for the whole algorithm if used propably for storing the solution path and then search for the implicit path. In this paper, we propose for an improved algorithm based on Dijkstra algorithm by using a special data structure (linked hash map) to store the solution path. The proposed algorithm is tested with different number of nodes. Results are recorded to justify the Algorithm validity.

\section{THE PROPOSED ALGORITHM DESCRIPTION}

The idea of Dijkstra algorithm is genius and amazing. In addition, it's simple and easy to understand especially by using non complicated data structure such as priority queue implemented as a min-heap. Although Fibonacci heap has achieved greater success and better performance, however, it's complicated and often theoretical more than practical issues [16]. Based on all of that, we use Dijkstra algorithm with priority queue implemented as a min-heap. For storing the solution path, we use a linked hash map within array list. This can give us a fast searching time equal to $\mathrm{O}(1)$ as an efficiency time. The following steps explain the main idea of Dijkstra algorithm [16]. 
DIJKSTRA. (G,w,s)

1 INITIALIZE-SINGLE-SOURCE.(G, s)

$2 \mathrm{~S}=\varnothing$;

$3 \mathrm{Q}=\mathrm{G} . \mathrm{V}$

4 while $\mathrm{Q} \neq \varnothing$;

$5 \mathrm{u}=$ EXTRACT-MIN $(\mathrm{Q})$

$6 \mathrm{~S}=\mathrm{SU}\{\mathrm{u}\}$

7 foreach vertex v $€$ G.Adj $[u]$

8 RELAX $(\mathrm{u}, \mathrm{v}, \mathrm{w})$

in step 1, the initialization of the source node $s$ in the graph $G$ is carried out. Step 2 initializes the set $\mathrm{S}$ to the empty set. The algorithm maintains the invariant that $\mathrm{Q}=\mathrm{V}-\mathrm{S}$ at the start of each iteration of the while loop of step 4 until step 8 . Step 3 initializes the min-priority queue Q to contain all the vertices in $\mathrm{V}$; since $\mathrm{S}=\varnothing$ at that time, the invariant is true after step 3. Each time through the while loop of steps 4 until step 8 , step 5 extracts a vertex $\mathrm{u}$ from $\mathrm{Q}=\mathrm{V}-\mathrm{S}$ and step 6 adds it to set $\mathrm{S}$, for the first time through this loop, $\mathrm{u}=\mathrm{s}$. Vertex $\mathrm{u}$, therefore, has the smallest shortest path estimate of any vertex in V - S. Then, step 7 and step 8 relax each edge $(\mathrm{u}, \mathrm{v})$. Notice that $\mathrm{w}$ is the wieght of the given edge.

We have built the storage data structure and identified a variant of the type of array-list which contains of a set of linked-hash-map (each value within the array-list is actually linked-hash-map includes a particular path) to store any path that has been queried .The hash-map is used to store the nodes and the distance between them. Each hash-map consists of (Key, Value). Each node and adjacent is stored in the key, while the distance between them is stored in Value. The following code describes the idea of storing and searching operations of the storage data structure. //storing operation

public static ArrayList $<$ LinkedHashMap $<$ Integer,Integer $>>$ AddtoPaths $($ List $<$ Integer $>$ path,int srcc,int destt)

\{

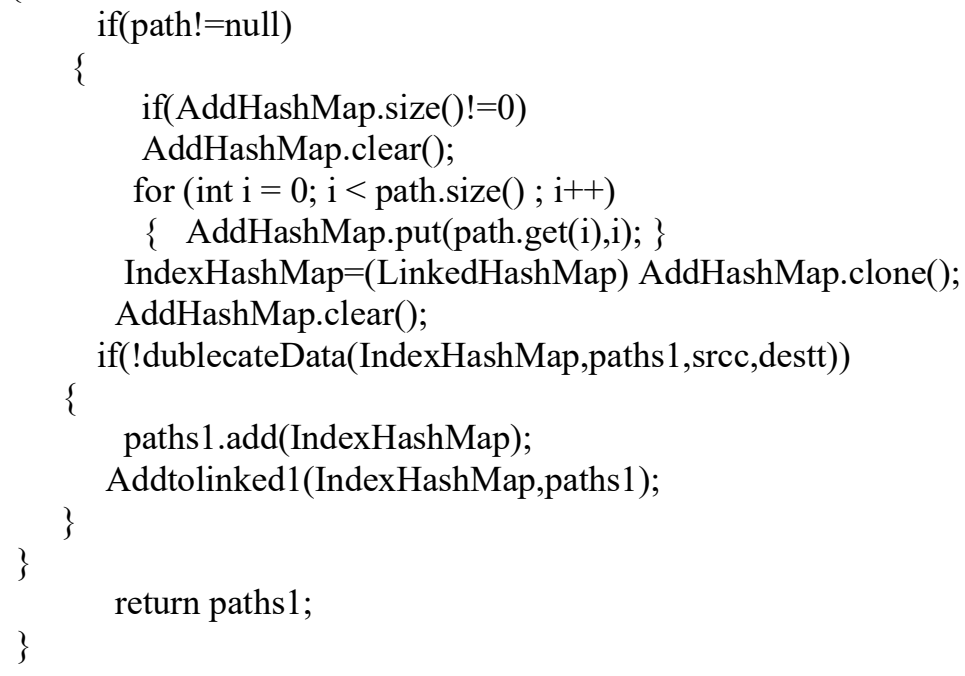

public static boolean dublecateData(LinkedHashMap $<$ Integer,

Integer $>$ IndexHashMap1,ArrayList $<$ LinkedHashMap $<$ Integer, Integer $>>$ paths11,int srcc1,int destt1)\{ for(LinkedHashMap<Integer, Integer $>$ p:paths 11) \{

if(p.equals(IndexHashMap1)) \{

return true; 
\}

for(LinkedHashMap $<$ Integer, Integer $>$ s:paths11)

\{ if( s.containsKey(srcc1) \&\& s.containsKey(destt1) ) \{ return true; \} \}

return false;

\}

public static void Addtolinked1(LinkedHashMap $<$ Integer,Integer $>$

path,ArrayList $<$ LinkedHashMap $<$ Integer,Integer $>>$ paths1) \{

int $\mathrm{ii}=1$;

int $\mathrm{i}$;

if( paths1.size ()$>0)\{$

$\mathrm{i}=$ paths1.size ()$-1$;

\}else $\{\mathrm{i}=$ paths $1 . \operatorname{size}() ;\}$

if(path!=null) \{

comp $=$ new ArrayList $<$ Integer $>$ (path.keySet ()$)$;

for (int $\mathrm{j}=0 ; \mathrm{j}<$ comp.size ()$; \mathrm{j}++)\{$

if(Number_of_Nodes.containsKey(comp.get(j)) \&\& Number_of_Nodes.get(comp.get(j))!=null

$\& \& \quad$ Number_of_Nodes.get(comp.get(j)).size ()$>0)$

\{

Number of Nodes.get(comp.get(j)).add(i);

\}else \{

Number_of_Nodes.put(comp.get(j),new ArrayList $<$ Integer $>())$;

\}

Number_of_Nodes.get(comp.get(j)).add(i);

\} $\}$

\} comp.clear();

//searching operation

public static ArrayList findShortestPathsDS(int src,int dest,Graph graph)

\{ start_time2 = System.nanoTime();

ArrayList subPath $=$ new ArrayList();

Number_of_Nodes

From=Number_of_Nodes.get(src);

To=Number_of_Nodes.get(dest);

if(MainClass.From! $!=$ null \&\& MainClass.To!=null \&\& MainClass.From.size ()$>0$ \& \& MainClass.To.size ()$>0)$

\{

\} else

$\{$ time $11=0.0$;

text = "\n The path is not implicit, So The path will be calculated using Dijkstra"; subPath $=$ (ArrayList)graph. findShortestPaths(src, dest);

end_time $2=$ System.nanoTime();

difference $2=($ end_time 2 - start_time2 $) / 1$ e6;

time11=Graph.time;

return subPath;

\}

$\operatorname{if}(\mathrm{SO}>=0)$

\{ 


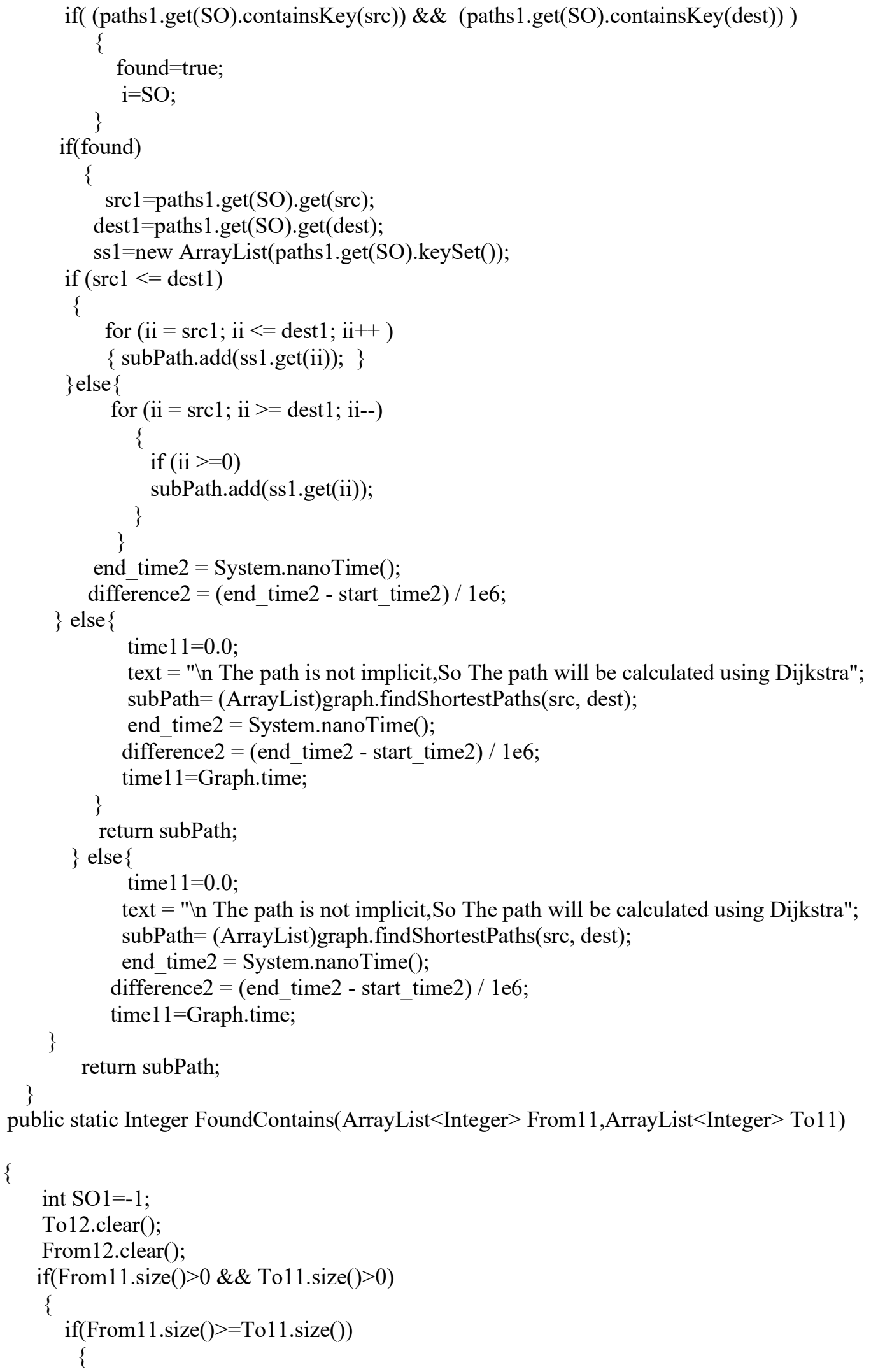




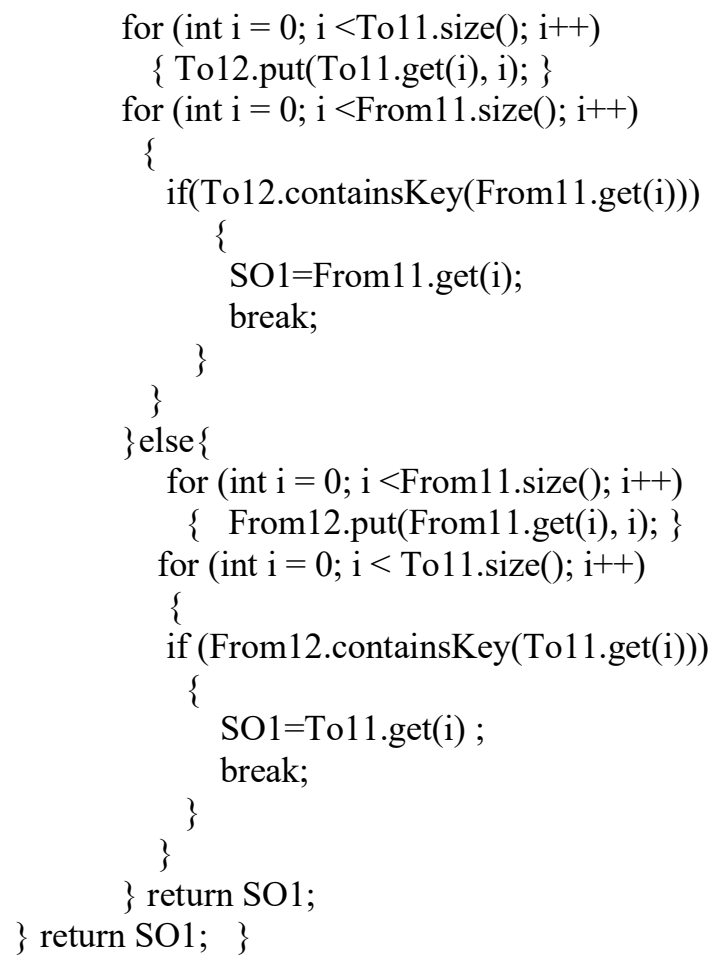

\section{THE ALGORITHM ANALYSIS AND RESULTS}

The time complexity of hash-map, linked-hash-map and array-list are different according to the kind of operations. Table 1 and 2 describes the time for each given data structure.

Table 1. The time complexity of hash-map and linked-hash-map

\begin{tabular}{|c|c|c|c|c|}
\hline & get & Contains Key & next & note \\
\hline Hash-map & $\mathrm{O}(1)$ & $\mathrm{O}(1)$ & $\mathrm{O}(\mathrm{h} / \mathrm{n})$ & $\mathrm{h}$ is the table capacity \\
\hline Linked-hash-map & $\mathrm{O}(1)$ & $\mathrm{O}(1)$ & $\mathrm{O}(1)$ & \\
\hline
\end{tabular}

Table 2. The time complexity of array-list

\begin{tabular}{|c|c|c|c|c|c|c|}
\hline & get & add & contains & next & remove & Iterator_remove \\
\hline Array-list & $\mathrm{O}(1)$ & $\mathrm{O}(1)$ & $\mathrm{O}(\mathrm{n})$ & $\mathrm{O}(1)$ & $\mathrm{O}(\mathrm{n})$ & $\mathrm{O}(\mathrm{n})$ \\
\hline
\end{tabular}

According to the previous tables, $\mathrm{n}$ means the number of nodes $(\mathrm{V})$, and $\mathrm{m}$ means the number of edges (E). The total time for putting the new solution path in the given data structure is:

$\mathrm{O}(\mathrm{n} \log n)+\mathrm{O}(\mathrm{n})+\mathrm{O}(\mathrm{n} \log n)=\mathrm{O}(\mathrm{n})+\mathrm{O}(2 \mathrm{n} \log n)=\mathrm{O}(2 \mathrm{n} \log n)=\mathrm{O}(\mathrm{n} \log n)$

In the other side, the total time complexity of the query from the data structure is:

$\mathrm{O}(\log n)+\mathrm{O}(\log n)+\mathrm{O}(\mathrm{n} \log n)+\mathrm{O}(\log n)+\mathrm{O}(\log n)$

$=\mathrm{O}(4 \log n)+\mathrm{O}($ nlogn $)$

$=\mathrm{O}(\log n)+\mathrm{O}(\mathrm{n} \log n)$

$=\mathrm{O}((1+\mathrm{n}) \log \mathrm{n})=\mathrm{O}(\mathrm{n} \log \mathrm{n})$

We run the original Dijkstra algorithm and the proposed algorithm with directed graph; where there is only one way from a node to other. We also run both of them with different number of 
nodes in order to get the shortest path between a given source node and destination. After the solution path is found, it's stored in the given data structure (array-list of linked-hash-map). Then, an inquiry for the implicit path is taken place. If the implicit path is found within the stored solution path in the given data structure, the time is calculated and recorded, else; the searching using the original Dijkstra algorithm is started again and the total time is recorded. The averages of each recorded times are calculated. These operations are repeated many times with different number of nodes. Table 3 and 4 contain the averages values of the time when the required path is the implicit path of the stored solution path and figure 1 and 2 shows the results analysis.

Table 3. The run time of the implicit path (directed graph)

\begin{tabular}{|c|c|c|}
\hline No. of nodes & $\begin{array}{c}\text { Dijkstra using only min-heap } \\
\text { Time average }\end{array}$ & $\begin{array}{c}\text { Dijkstra using min-heap with } \\
\text { ArrayList }<\text { LinkedHashMap }> \\
\text { Time average }\end{array}$ \\
\hline 100 & 0.66256 & 0.28489 \\
\hline 200 & 1.47381 & 0.3076 \\
\hline 300 & 2.06859 & 0.34078 \\
\hline 400 & 4.15724 & 0.38133 \\
\hline 500 & 5.56397 & 0.28971 \\
\hline 600 & 2.552 & 0.24657 \\
\hline 700 & 3.15004 & 0.51842 \\
\hline 800 & 8.35776 & 0.22292 \\
\hline 900 & 5.11631 & 0.21436 \\
\hline 1000 & 4.81021 & 0.2146 \\
\hline
\end{tabular}

\section{IMPLICIT}

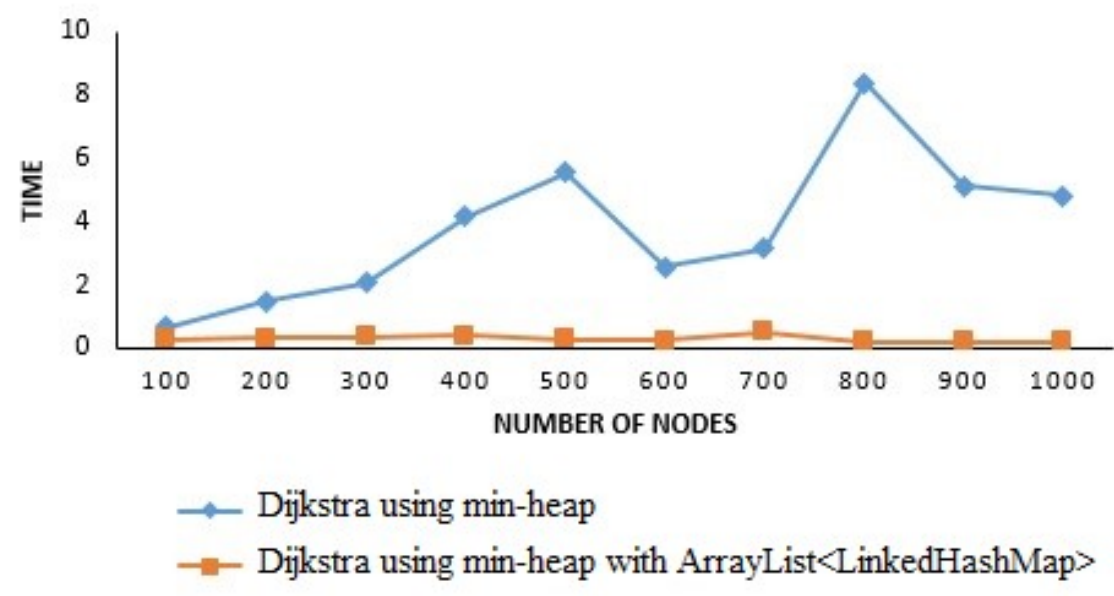

Figure 1. The run time of 100-1000 number of nodes (directed graph)

From figure 1, we notice that the time decreases as the number of nodes increases. Time average is equal to 0.28489 when the number of nodes is 100 , then time increases with unobserved amount. It gives the highest value when 700 nodes and starts to decrease at 800 nodes and above. 
Table 4. The run time of the implicit path (directed graph)

\begin{tabular}{|c|c|c|}
\hline No. of nodes & $\begin{array}{c}\text { Dijkstra using only min-heap } \\
\text { Time average }\end{array}$ & $\begin{array}{c}\text { Dijkstra using min-heap with } \\
\text { ArrayList }<\text { LinkedHashMap }> \\
\text { Time average }\end{array}$ \\
\hline 1000 & 4.81021 & 0.2146 \\
\hline 2000 & 11.03552 & 0.18769 \\
\hline 3000 & 13.76008 & 0.17272 \\
\hline 4000 & 12.66174 & 0.19816 \\
\hline 5000 & 15.29122 & 0.16887 \\
\hline 6000 & 20.46555 & 0.18637 \\
\hline 7000 & 47.87112 & 0.1797 \\
\hline 8000 & 27.05117 & 0.17704 \\
\hline 9000 & 55.55694 & 0.18778 \\
\hline 10000 & 42.26821 & 0.17102 \\
\hline
\end{tabular}

IMPLICIT

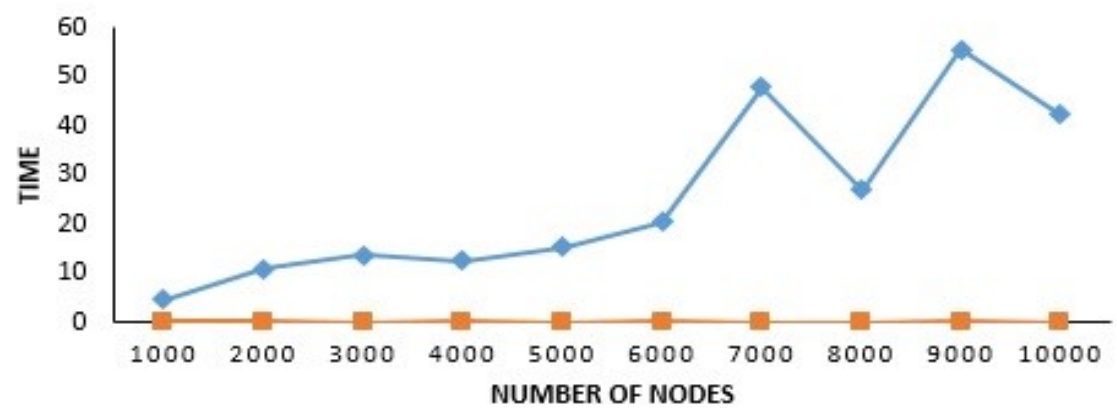

$\rightarrow$ Dijkstra using min-heap

Dijkstra using min-heap with ArrayList $<$ LinkedHashMap >

Figure 2. The run time of 1000-10000 numbers of nodes (directed graph)

Looking at figure 2, nodes numbers are various from 1000 to 10000 nodes. We realize that the time decreases as the number of nodes increase and that improved the proposed algorithm validity. We observe a linear time for the given data structure; ArrayList $<$ LinkedHashMap $>$.

Table 5 and 6 contain the averages values of the time when the required path is not the implicit path of the stored solution path and figure 3 and 4 show the results analysis.

Table 5. The run time of the non-implicit path (directed graph)

\begin{tabular}{|c|c|c|}
\hline No. of nodes & $\begin{array}{c}\text { Dijkstra using by } \\
\text { min-heap }\end{array}$ & $\begin{array}{c}\text { Dijkstra using by min-heap with } \\
\text { ArrayList }<\text { LinkedHashMap }>\end{array}$ \\
\hline 100 & 0.86681 & 0.92647 \\
\hline 200 & 2.30324 & 2.41939 \\
\hline 300 & 3.15914 & 3.27483 \\
\hline 400 & 3.45716 & 3.54365 \\
\hline 500 & 4.5522 & 4.64095 \\
\hline 600 & 4.08183 & 4.17757 \\
\hline 700 & 7.1498 & 7.26413 \\
\hline 800 & 5.8222 & 5.90921 \\
\hline 900 & 7.55844 & 7.65247 \\
\hline 1000 & 7.73631 & 7.84603 \\
\hline
\end{tabular}




\section{NON IMPLICIT}

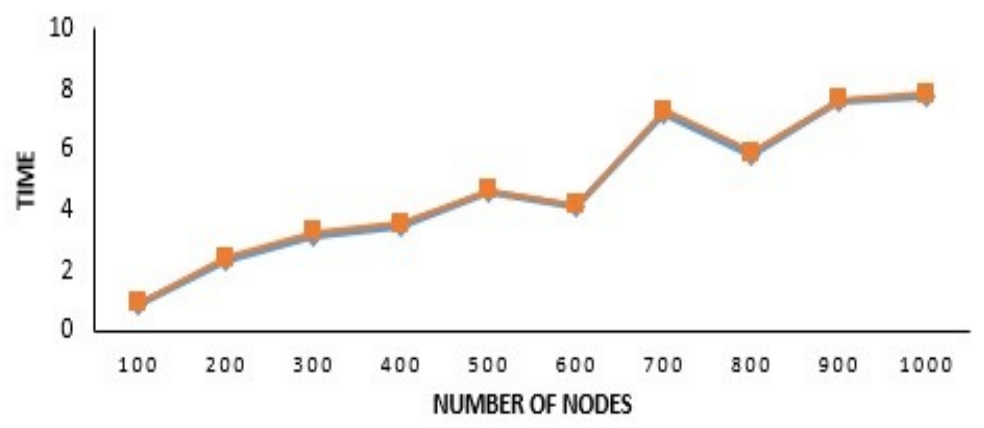

$\rightarrow$ Dijkstra algorithm using min-heap

$\rightarrow$ Dijkstra algorithm using min-heap with ArrayList<LinkedHashMap >

Figure 3. The run time of 100-1000 number of nodes (directed graph)

Figure 3 describes the run time of non-implicit path if it's not included within the stored solution shortest path. The relationship between run time and nodes numbers is shown. It is observed that the time for the proposed algorithm is almost equal to the original Dijkstra algorithm with minheap. There is a slightly fixed difference as the nodes numbers increases.

Table 6. The run time of the non-implicit path (directed graph)

\begin{tabular}{|l|l|l|}
\hline No. of nodes & $\begin{array}{l}\text { Dijkstra using by min } \\
\text { heap }\end{array}$ & $\begin{array}{l}\text { Dijkstra using by min heap with } \\
\text { ArrayList }<\text { LinkedHashMap }>\end{array}$ \\
\hline 1000 & 7.73631 & 7.84603 \\
\hline 2000 & 11.90012 & 12.02769 \\
\hline 3000 & 17.38345 & 17.50731 \\
\hline 4000 & 31.68681 & 31.79614 \\
\hline 5000 & 35.59574 & 35.68865 \\
\hline 6000 & 57.20521 & 57.31768 \\
\hline 7000 & 67.07719 & 67.16318 \\
\hline 8000 & 35.34394 & 35.4589 \\
\hline 9000 & 73.9846 & 74.109 \\
\hline 10000 & 69.43308 & 69.54271 \\
\hline
\end{tabular}

\section{NON IMPLICIT}

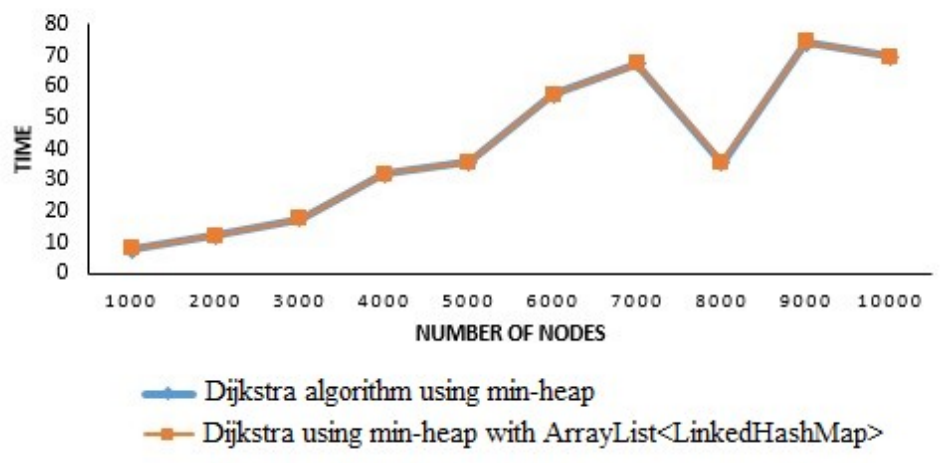

Figure 4. The run time of 1000-10000 number of nodes (directed graph) 
From figure 3 and 4, we notice that almost the same time is observed for searching for a path which is not a sub path from the solution shortest path.

\section{DISCUSSION}

In this paper, a case study of random directed graph with different number of nodes has been carried out in order to test the validity of our proposed algorithm. The same case study has been given to the original Dijkstra algorithm with priority queue implemented as a min-heap. The results of both algorithms have been recorded and analysed. Comparisons between these results have shown that the proposed algorithm is almost the best. Although more data structures have been used within the proposed algorithm, however, the enlarged storage is available for all of the current devices, even for the smallest one. We argue that data storages aren't problem if the performance of the given algorithm is higher and success. It has been observed that the searching time of the original algorithm is almost the same as the time of the proposed algorithm if the required path is not an implicit path within the solution path. Regarding of the results in the literature, run time is various depending on the type of used data structure and also CPU speed. Although our results give a good time average for searching within a solution path, we realize that the greater the number of nodes, time becomes less and less. This can work in a large storage of nodes especially in a huge road network.

\section{CONCLUSION AND FUTURE WORK}

The analysis of searching time for implicit path seems to be rare and less concern. Most available algorithms expect the nature of easy time searching for sub path within the solution path. Well, data structure plays main role in the whole procedures and operations. We realize this point when we start proposing our idea for improvement of the available Dijkstra algorithm. We consider improving the time complexity of implicit path within the solution path as a first starting point. According to our results, the improvement of the proposed algorithm is achieved with regard of directed graph. This type of graph could reflect the one way road in reality. The proposed algorithm achieves the best result especially for a large number of nodes. Our future work will consider the undirected graph. We also plan to apply our proposed algorithm to a real network road map to show the performance and improve the validity from a real point of view.

\section{REFERENCES}

[1] Arman, N., \& Khamayseh, F., (2015) "A Path-Compression Approach for Improving Shortest-Path Algorithms," International Journal of Electrical and Computer Engineering, vol. 5, p. 772,

[2] Broumi, S., Bakal, A., Talea, M., Smarandache, F., \& Vladareanu, L., "Applying Dijkstra algorithm for solving neutrosophic shortest path problem," International Conference on Advanced Mechatronic Systems (ICAMechS), 2016, pp. 412-416.

[3] Gao, J., Zhao, Q., Ren, W., Swami, A., Ramanathan, R., \& Bar-Noy, A., (2015) "Dynamic shortest path algorithms for hypergraphs," IEEE/ACM Transactions on Networking, vol. 23, pp. 1805-1817.

[4] Gutenschwager, K., Völker, S., Radtke, A., \& Zeller, G., "The shortest path: Comparison of different approaches and implementations for the automatic routing of vehicles," in Simulation Conference (WSC), Proceedings of the 2012 Winter, 2012, pp. 1-12.

[5] Khamayseh, F., \& Arman, N., (2015) "Improvement of Shortest-Path Algorithms Using Subgraph's Heuristics". Journal of Theoretical \& Applied Information Technology, vol. 76. 
[6] Niemeyer, K., E., \& Sung, C.-J., (2016) "On the importance of graph search algorithms for DRGEPbased mechanism reduction methods," Combustion and Flame, vol. 158, pp. 1439-1443.

[7] Shu-Xi, W., (2012) "The improved dijkstra's shortest path algorithm and its application," Procedia Engineering, vol. 29, pp. 1186-1190.

[8] Douglas,W., B., (2001) Introduction to Graph Theory, Pernice Hall.

[9] Chandra, "Shortest Path Problem for Public Transportation Using GPS and Map Service," 2012.

[10] Saab, Y., \& VanPutte, M., (1999) "Shortest path planning on topographical maps," IEEE Transactions on Systems, Man, and Cybernetics-Part A: Systems and Humans, vol. 29, pp. 139-150.

[11] Xing, S., \& Shahabi, C., "Scalable shortest paths browsing on land surface," in Proceedings of the 18th SIGSPATIAL International Conference on Advances in Geographic Information Systems, 2010 , pp. 89-98.

[12] Faro, A., \& Giordano, D., (2016) "Algorithms to find shortest and alternative paths in free flow and congested traffic regimes," Transportation Research Part C: Emerging Technologies, vol. 73, pp. 129.

[13] Dijkstra., E., W., (1959) "A note on Two Problems in Connexion with graphs", Numerische Mathematik, 1, 269-271.

[14] Thorat, S., \& Rahane, S., (2016) "Review of Shortest Path Algorithm”, IRJET, vol 3, issue 8.

[15] Yao, B., Yin, J., Zhou, H., \& Wu, W., (2016) "Path Optimization Algorithms Based on Graph Theory," International Journal of Grid and Distributed Computing, vol. 9, pp. 137-148.

[16] Cormen, T., Leiserson, C., Rivest, R., \& Stein, C., (2009) Introduction to Algorithms, 3rd. ed., MIT Press, London.

[17] Levitin, A., (2012) Introduction to the Design and Analysis of Algorithms, 3rd ed., Pearson Education, Inc., Addison-Wesley.

[18] Jain, A., Datta, U., \& Joshi, N., (2016) "Implemented modification in Dijkstra ${ }^{\text {ee }}$ s Algorithm to find the shortest path for $\mathrm{N}$ nodes with constraint," International Journal of Scientific Engineering and Applied Science, vol. 2, pp. 420-426.

[19] Xie, D., Zhu, H., Yan, L., Yuan, S., \& Zhang, J., "An improved Dijkstra algorithm in GIS application," in World Automation Congress (WAC), 2012, pp. 167-169.

[20] Lu, J., \& Dong, C., "Research of shortest path algorithm based on the data structure," in the 3rd International Conference of Software Engineering and Service Science (ICSESS), 2012 IEEE, 2012, pp. 108-110.

[21] Kong, D., Liang, Y., Ma, X., \& Zhang, L., "Improvement and Realization of Dijkstra Algorithm in GIS of Depot," in the International Conference on Control, Automation and Systems Engineering (CASE), 2011, 2011, pp. 1-4.

[22] Deng, Y., Chen, Y., Zhang, Y., \& Mahadevan, S., (2012) "Fuzzy Dijkstra algorithm for shortest path problem under uncertain environment," Applied Soft Computing, vol. 12, pp. 1231-1237. 


\section{AUTHORS}

Mabroukah Amarif: received her BSc degree in Computer Science from University of Sebha, Libya, MSc in Computer Science from Universiti Sains Malaysia, and PhD in Software Engineering from Universiti Kebangsaan Malaysia. Her interests span a wide range of topics in the area of Software Engineering, Networking, Computer Security, Visual Informatic, Computer Education and programming languages. She is currently working as Assistant Professor at the departement of computer science, Faculty of Information Technology in Sebha University of Libya.

Ibtusam Alashoury: received her BSc degree in Computer Science from University of Sebha, Libya. She is currently doing her MSc in computer Sciences at Sebha University of Libya. She interests in the area of Software Engineering, System Analysis and Web design. she is currently working as a technical Engineer in the information development project of Sebha University of Libya.
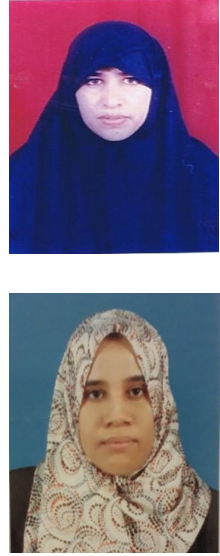